\title{
Rectangular renormalization
}

\author{
Jeffrey I Prentis $†$ and Bruce S Elenbogenł \\ † Department of Natural Sciences, University of Michigan-Dearborn, Dearborn, MI 48128, USA \\ $\ddagger$ Department of Computer and Information Sciences, University of Michigan-Dearborn, \\ Dearborn, MI 48128, USA
}

Received 6 June 1995

\begin{abstract}
A generalized real-space renormalization scheme is developed for geometrical critical phenomena. The renormalization group is parametrized by the standard length-scaling factor and a new rectangular area-fraction factor. This rectangular renormalization scheme utilizes relatively small rectangular sublattices to effectively renormalize large square lattices. With the area-fraction factor, one can systematically study rectangular generalizations of the conventional square-cell renormalization theories. Application to self-avoiding random walks yields critical descriptors that are comparable to, and in most cases better than previous results obtained from more complex renormalization schemes.
\end{abstract}

\section{Introduction}

The renormalization-group theory is a vital tool to understanding critical phenomena. The real-space formulation of the renormalization group, by virtue of its simplicity and versatility, is widely used to study geometrical and thermodynamical systems [1]. Recent applications include surface phenomena [2], and self-organized criticality, such as diffusionlimited aggregation and sandpiles [3].

In spite of its ubiquitous role in the theory of critical phenomena and scale-invariant systems, the practical implementation of real-space renormalization remains somewhat of an art, rather than a science. This is due to the uncontrolled nature of the approximations that are inherent to the real-space formulation. This is in contrast to field theory, or momentumspace renormalization for which there exists a small parameter for systematically controlling a perturbation expansion. In real-space renormalization, there does not exist any natural small parameter or rigorous set of criteria to ascertain a priori whether or not a given approximation will yield results that are accurate and/or convergent. For geometrical critical phenomena defined on lattices, all the approximations involve some type of truncation to a finite lattice, together with a rule for renormalization of the degrees of freedom. In spite of these seemingly drastic approximations, it is fortuitous that one can obtain reasonable results for the critical behaviour, usually within $5 \%$ to $10 \%$ of the exact values. However, in order to improve the accuracy, one must rely on more intricate techniques and additional rules. This can add substantial complexity to the basic 'zeroth-order renormalization scheme. This higher-order complexity includes the use of additional couplings, transfer matrices, toroidal lattices, Monte Carlo algorithms, finite-size scaling and other extrapolation methods to extend the small lattice results to larger lattices [4]. It is unfortunate that this large addition of technical complexity is usually incommensurate with the small gain in numerical accuracy. As a further complication, there exits a fundamental pathology of the real-space 
renormalization-group theory that renders it problematic in certain cases [1,5]. A peculiar inconsistency in the standard real-space renormalization theory of percolation has recently been uncovered [6,7].

In this paper, we present a generalized real-space renormalization scheme for geometrical critical phenomena that contains some of the conventional schemes as special cases. This generalized or rectangular renormalization represents a simple zeroth-order renormalization scheme, free of the complexities that characterize the higher-order methodology. Application of rectangular renormalization to the self-avoiding random walk problem in two dimensions yields critical points and exponents that are accurate, stable and convergent. In particular, simple calculations on small rectangular cells can produce results that are better than those obtained using large square cells, multiple cell clusters, and other more sophisticated renormalization schemes.

\section{Renormalization}

Consider any geometrical critical phenomena where the connectivity of the fluctuating degrees of freedom is the microscopic trademark responsible for the long-range correlations, or scale invariance. The prototype geometrical systems, or lattice statistics problems, that exhibit critical behaviour are the self-avoiding random walk and percolation. For concreteness, we shall take the self-avoiding random walk on a lattice to illustrate the renormalization.

The renormalization-group theory allows one to understand the critical behaviour by probing the system at different length scales. The renormalization transformation is the equation that exhibits the structure of the system at these different length scales. In the real-space realization of the renormalization group, the basic definition [1] of the renormalization map is

$$
W^{\prime}\left(s^{\prime}\right) \equiv \sum_{s} P\left(s^{\prime}, s\right) W(s)
$$

where $W(s)$ is the statistical weight (Boltzmann factor) of a state $s$ on the original lattice, and $W^{\prime}\left(s^{\prime}\right)$ is the statistical weight of a renormalized (coarse-grained) state $s^{\prime}$ on the rescaled lattice. The rescaled lattice is isomorphic to the original lattice and has a larger lattice spacing. The projection matrix $P\left(s^{\prime}, s\right)$ specifies how to project the object state $s$ onto the image state $s^{\prime}$. More specifically, $P\left(s^{t}, s\right)$ determines the conditional probability that $s^{\prime}$ is the image of $s$ under the renormalization. The only constraint imposed on the projection matrix is that it must satisfy

$$
\sum_{s^{\prime}} P\left(s^{\prime}, s\right)=1 \text {. }
$$

This normalization condition insures that the partition function (sum-over-states) is a renormalization-group invariant:

$$
\sum_{s^{\prime}} W^{\prime}\left(s^{\prime}\right)=\sum_{s} W(s)
$$

Hence average values (observables) generated from the partition function will be identical in the object and image spaces.

For the purpose of formulating a generalized renormalization scheme, we classify current real-space renormalization schemes into two categories:

(i) 'Single-state' renormalization: the renormalization map is computed directly from (2.1) by focusing on one renormalized state $s^{\prime}$ at a time and the corresponding weight $W^{\prime}\left(s^{\prime}\right)$. To compute $W^{\prime}\left(s^{\prime}\right)$, a rule must be formulated that determines the subset of object states compatible with the single image state $s^{\prime}$. The cell-to-bond [8], site decimation [9], and cumulant [10] renormalization schemes fall into this category. 

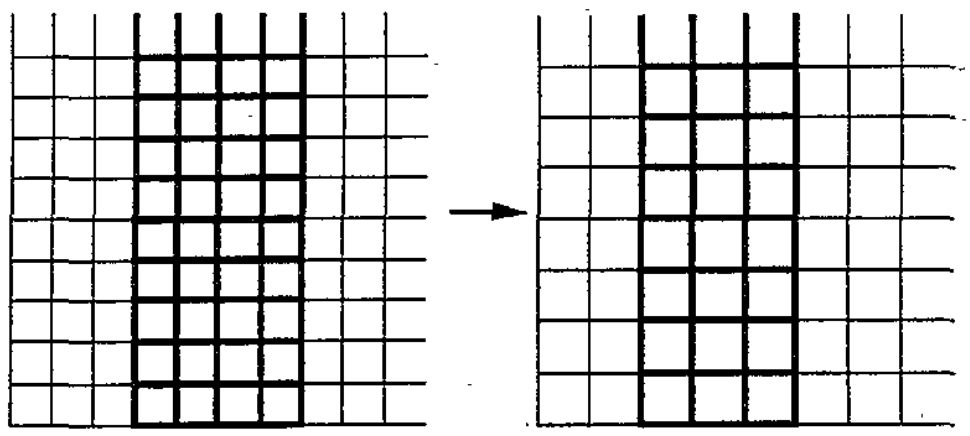

Figure 1. The mapping between the $\Omega$-lattices, $10 \times 10 \rightarrow 8 \times 8$, and between the rectangular $\omega$-lattices, $10 \times 5 \rightarrow 8 \times 4$ (in bold), corresponding to a renormalization with length factor $b=\frac{5}{4}$ and area factor $f=\frac{1}{2}$.

(ii) 'All-state' renormalization. The renormalization map is computed from (2.3) by focusing on the set of all renormalized states simultaneously and the corresponding weight for the whole set of states. Note that this all-state weight is the sum-over-states of the singlestate weights, or the whole partition function. The cell-to-cell renormalization scheme [8] falls into this category.

In what follows, we formulate a more general renormalization scheme based on a partial partition function corresponding to a partial set of states on a rectangular sub-]attice. Consider a finite square lattice, denoted by $\Omega$, of size $n \times n$, where $n$ is the number of bonds on a side. Each bond is of length $\ell$. The rescaled square lattice, denoted $\Omega^{\prime}$, has a size $n^{\prime} \times n^{\prime}$ and a bond length $\ell^{\prime}$. The two microscopic length scales, $\ell$ and $\ell^{\prime}$, are related by the scaling factor $b$ defined by

$$
\ell^{\prime}=b \ell
$$

where $1<b<\infty$. The original and the rescaled finite lattices, shown in figure 1 , each represent one truncated cell of the infinite square lattice. This truncated square cell characterizes the single-cell renormalization schernes [8]. The collection of such square cells completely tile the infinite (parent) square lattice.

Now consider a rectangular sub-cell, denoted by $\omega$, of the original square cell, and the corresponding rectangular sub-cell, denoted $\omega^{\prime}$, of the rescaled square cell (see figure 1). To symbolically indicate that the rectangular sub-cell area is a fraction of the square cell area, we write

$$
\omega=f \Omega \quad \text { and } \quad \omega^{\prime}=f \Omega^{\prime}
$$

where $f$ is the area fraction factor that can take on the values $0<f \leqslant 1$. We define the renormalization transformation by mapping the set of object states confined to the $\omega$-lattice onto the image states confined to the $\omega^{\prime}$-lattice. Both object and image states should span the rectangular region by connecting the bottom edge to the top edge of the rectangle. This spanning rule is natural and common [4]. It preserves the essential long-range or macroscopic connectivity of the degrees of freedom.

Thus our renormalization is effectively a map between spanning states on a rectangular sub-lattice of size $n \times f n$ and spanning states on a rectangular sub-lattice of size $n^{\prime} \times f n^{\prime}$. For the special case $f=1$, we recover the frequently used cell-to-bond $\left(n^{\prime}=1\right)$ and cell-tocell $\left(n^{\prime} \neq 1\right)$ renormalization schemes [8]. Note that $f=1 / n^{\prime}$ corresponds to a single-state renormalization scheme which is a generalized cell-to- 'bond' mapping: $n \times\left(n / n^{\prime}\right) \rightarrow n^{\prime} \times 1$. 
Given the additional freedom afforded by the area fraction factor $f$, that can assume a continuum of values between 0 and 1 , one can explore a new class of renormalizations.

In more formal terminology, rectangular renormalization is defined by a projection matrix $P\left(s^{\prime}, s\right)$ that preserves the rectangular area spanned by the object state $s$ and the image state $s^{\prime}$. That is, $P\left(s^{\prime}, s\right)$ takes on the value 1 if $s$ and $s^{\prime}$ are confined to the same rectangular area, and is 0 otherwise. A less restrictive definition of the projection matrix is

$$
\sum_{s^{\prime} \in \omega^{\prime}} P\left(s^{\prime}, s\right)= \begin{cases}1 & s \in \omega \\ 0 & s \notin \omega\end{cases}
$$

where $s \in \omega\left(s^{\prime} \in \omega\right)$ denotes those spanning states confined to, or completely embedded in the original rectangular lattice $\omega$ (rescaled rectangular lattice $\omega^{\prime}$ ). Those spanning states that are not confined to the $\omega$-lattice are denoted by $s \notin \omega$.

This choice of projection matrix is physically reasonable. In addition to preserving the long-range connectivity of the states, via spanning the rectangle, it also preserves the overall topology or coarse-grained shape of the states, by confining them to the same rectangular region. Each spanning state sweeps out a rectangular region as it spans the cell. The renormalization is a mapping between those spanning states whose bounding rectangular area is equal to or less than the area of $\omega$.

In other words, the rectangular $\omega$-lattice represents an 'active zone' and the complement of $\omega$ is the 'forbidden zone'. Spanning states with degrees of freedom that venture into the forbidden zone have zero projection to the active zone. Neglecting states that partially visit the forbidden zone is an approximation that is made for the sake of simplicity to avoid additional, arbitrary nules. It corresponds to zeroth-order "perturbation theory". As the cell size increases, this approximation is expected to become more exact since these partially forbidden states represent a perturbation to the active states. The fraction of these perturbed states decreases as the cell size increases. Our results support this expected surface effect. The binary classification of macroscopic spanning states into active-zone states or forbiddenzone states is a geometric analogue of Ising spin states in a magnetic system which can have the values of up or down. This is especially evident for the case $f=\frac{1}{2}$ for which the active and forbidden zones have equal area. As far as the renormalization (projection matrix) is concerned, there exist only two kinds of spanning states: active or forbidden.

Given the area-preserving projection matrix, the rectangular renormalization transformation assumes the form

$$
\sum_{s^{\prime} \in f \Omega^{\prime}} W^{\prime}\left(s^{\prime}\right)=\sum_{s \in f \Omega} W(s)
$$

This map follows from the basic renormalization equation (2.1) together with our definition of the rectangular projection matrix in (2.6). Note that we have also used $\omega=f \Omega$ and $\omega^{\prime}=f \Omega^{\prime}$ in the sum-over-states to indicate the explicit dependence of this map on the area fraction factor $f$.

An alternative insight into the formal structure of rectangular renormalization can be found upon dissecting the invariant partition function relation in (2.3). Consider the decomposition of the sum-over-states into two partial sums:

$$
\sum_{s^{\prime} \in \omega^{\prime}} W^{\prime}\left(s^{\prime}\right)+\sum_{s^{\prime} \notin \omega^{\prime}} W^{\prime}\left(s^{\prime}\right)=\sum_{s \in \omega} W(s)+\sum_{s \notin \omega} W(s) .
$$

The rectangular renormalization map corresponds to the equality of the first term on each side of this equation. This first term corresponds to the partial partition function of the active zone states. Hence, the whole partition function is invariant via the separate invariance of partial partition functions. 
It is instructive to compare the generalized, rectangular renormalization equation (2.7) to existing renormalization equations using the symbolism developed in this paper. The singlestate (cell-to-bond, site decimation, cumulant) renormalization equation assumes the form

$$
W^{\prime}\left(s^{\prime}\right)=\sum_{s \in \Omega} P\left(s^{\prime}, s\right) W(s)
$$

In this expression, $\Omega$ represents any truncated lattice, including a single cell, a multiple-cell cluster, or a decimated lattice. The all-state (cell-to-cell) renormalization equation assumes the form

$$
\sum_{s^{\prime} \in \Omega^{\prime}} W^{\prime}\left(s^{\prime}\right)=\sum_{s \in \Omega} W(s)
$$

When compared with the generalized renormalization equation $(2.7)$, these other equations are characterized by $f=1$, i.e. a sum over all states on the truncated lattice. An advantage of the all-state renormalization scheme is that it does not require a detailed specification of the projection matrix. In general, the all-state renormalization schemes give more accurate results than the single-state schemes [4]. This could be due to the fact that by only focusing on a single renormalized state, the invariance of the whole partition function is more easily violated.

\section{Application to self-avoiding random walks}

We apply rectangular renormalization to the self-avoiding random walk in two dimensions. This critical system serves as a paradigm for geometrical critical phenomena. For a selfavoiding random walk of $N$ steps, the critical behaviour is characterized by the scaling of the multiplicity function (entropy) and the average size (correlations) of the walks for large $N$ :

$$
\begin{aligned}
& Z \sim \mu^{N} N^{\gamma-1} \\
& R \sim N^{v}
\end{aligned}
$$

where $Z$ is the total number of walks and $R$ is the root-mean-squared end-to-end distance of the walks. The critical exponents $\gamma$ and $v$ determine the universal power law divergences, while the lattice connective constant $\mu$ determines the effective coordination number of the lattice. The critical descriptors, $\mu$ and $\nu$, can be obtained from the real-space renormalization map in the usual way [8]. In the grand canonical statistics, each nearest-neighbour step of the object walk is weighted with a fugacity $K$, and each step of the image walk is weighted with $K^{\prime}$. To maintain the simplicity of our scheme, we do not consider additional weights associated with steps beyond nearest-neighbour. Also for simplicity, we adopt the standard corner rule [8] for spanning the cell. Given the grand canonical statistical weight $W(s)=K^{N}$ associated with each $N$-step walk, the rectangular renormalization equation (2.7) becomes a one-dimensional polynomial map parametrized by the length scaling factor $b$ and the area fraction factor $f$. We denote this map by $K_{b f}^{\prime}(K)$. The non-trivial fixed point $K_{b f}^{*}$ of this nonlinear map and the eigenvalue $\lambda_{b f}$ of the linearized map determine the critical descriptors according to

$$
\begin{aligned}
\mu_{b f} & =\frac{1}{K_{b f}^{*}} & \text { where } \quad K_{b f}^{*} & =K^{\prime}\left(K_{b f}^{*}\right) \\
\nu_{b f} & =\frac{\ln b}{\ln \lambda_{b f}} . & \text { where } \quad \lambda_{b f}^{-} & =\frac{\mathrm{d} K^{\prime}}{\mathrm{d} K}\left(K_{b f}^{*}\right) .
\end{aligned}
$$

The results for the critical point $K^{*}$ and the critical exponent $v$ are displayed in tables 1-6 for various rectangular renormalizations. Note that the critical descriptors converge toward 
Table 1. The critical point and exponent for area fraction $f=\frac{1}{2}$.

\begin{tabular}{llllll}
\hline & & \multicolumn{5}{c}{$n^{\prime} \times \frac{1}{2} n^{\prime}$} \\
\cline { 3 - 6 }$n \times \frac{1}{2} n$ & & $2 \times 1$ & $4 \times 2$ & $6 \times 3$ & $8 \times 4$ \\
\hline $4 \times 2$ & $K^{*}$ & 0.4656 & & & \\
& $v$ & 0.7153 & & & \\
$6 \times 3$ & $K^{*}$ & 0.4422 & 0.4235 & & \\
& $v$ & 0.7225 & 0.7322 & & \\
$8 \times 4$ & $K^{*}$ & 0.4286 & 0.4148 & 0.4072 & \\
& $\nu$ & 0.7271 & 0.7363 & 0.7420 & \\
$10 \times 5$ & $K^{*}$ & 0.4198 & 0.4090 & 0.4029 & 0.3989 \\
& $v$ & 0.7300 & 0.7388 & 0.7440 & 0.7466 \\
\hline
\end{tabular}

Table 2. The critical point and exponent for area fraction $f=\frac{1}{3}$.

\begin{tabular}{lllll}
\hline & & \multicolumn{3}{c}{$n^{\prime} \times \frac{1}{3} n^{\prime}$} \\
\cline { 3 - 5 }$n \times \frac{1}{3} n$ & & $3 \times 1$ & $6 \times 2$ & $9 \times 3$ \\
\hline $6 \times 2$ & $K^{*}$ & 0.4656 & & \\
& $\nu$ & 0.7153 & & \\
$9 \times 3$ & $K^{*}$ & 0.4406 & 0.4206 & \\
& $\nu$ & 0.7241 & 0.7364 & \\
$12 \times 4$ & $K^{*}$ & 0.4265 & 0.4119 & 0.4042 \\
& $\nu$ & 0.7291 & 0.7404 & 0.7460 \\
\hline
\end{tabular}

Table 3. The critical point and exponent for area fraction $f=\frac{1}{4}$.

\begin{tabular}{llll}
\hline & & \multicolumn{2}{c}{$n^{3} \times \frac{1}{4} n^{\prime}$} \\
\cline { 2 - 4 }$n \times \frac{1}{4} n$ & & $4 \times 1$ & $8 \times 2$ \\
\hline $8 \times 2$ & $K^{*}$ & 0.4656 & \\
& $\nu$ & 0.7153 & \\
$12 \times 3$ & $K^{*}$ & 0.4398 & 0.4191 \\
& $v$ & 0.7249 & 0.7386 \\
\hline
\end{tabular}

Table 4. The critical point and exponent for area fraction $f=\frac{1}{3}$.

\begin{tabular}{llll}
\hline & & \multicolumn{2}{c}{$n^{\prime} \times \frac{1}{5} n^{\prime}$} \\
\cline { 3 - 4 }$n \times \frac{3}{3} n$ & & $5 \times 1$ & $10 \times 2$ \\
\hline $10 \times 2$ & $K^{*}$ & 0.4656 & - \\
& $\nu$ & 0.7153 & - \\
$15 \times 3$ & $K^{*}$ & 0.4393 & 0.4182 \\
& $\nu$ & 0.7253 & 0.7400 \\
\hline
\end{tabular}

the best known numerical estimate of $K^{*}=0.379052$ [11] and the exact value of $\nu=\frac{3}{4}$ [12] as $b \rightarrow \infty$ (down a column of the table) or as $b \rightarrow 1$ (across a row or down the diagonal of the table). This behaviour as a function of the length scaling factor $b$ for different area fractions $f$ is a generalization of the $b$-dependence observed in the conventional square-cell $(f=1$ ) renormalization schemes [8].

In table 7, we display the critical descriptors in the parameter space of the renormalization group. Each point $(b, f)$ in this space corresponds to a particular renormalization 
Table 5. The critical point and exponent for area fraction $f=\frac{1}{6}$.

\begin{tabular}{llll}
\hline & & \multicolumn{2}{c}{$n^{\prime} \times \frac{1}{6} n^{\prime}$} \\
\cline { 2 - 4 }$n \times \frac{1}{6} n$ & & $6 \times 1$ & $12 \times 2$ \\
\hline $12 \times 2$ & $K^{*}$ & 0.4656 & \\
& $v$ & 0.7153 & \\
$18 \times 3$ & $K^{*}$ & 0.4390 & 0.4176 \\
& $v$ & 0.7257 & 0.7408 \\
\hline
\end{tabular}

Table 6. The critical point and exponent for area fractions $f=\frac{2}{3}$ and $f=\frac{3}{4}$.

\begin{tabular}{llll}
\hline & & \multicolumn{2}{c}{$n^{\prime} \times f n^{\prime}$} \\
\cline { 2 - 3 }$n \times f n$ & & $3 \times 2$ & $4 \times 3$ \\
\hline $6 \times 4$ & $K^{*}$ & 0.4177 & \\
& $\nu$ & 0.7324 & \\
$8 \times 6$ & $K^{*}$ & & 0.4036 \\
& $v$ & & 0.7400 \\
\hline
\end{tabular}

Table 7. Critical descriptors in the parameter space $(b, f)$ of the rectangular renormalization group. For each length scaling factor $b$ and area fraction factor $f$, the critical point and exponent are displayed. The first four rows represent single-state (generalized cell-to-bond) renormalizations. The last four rows represent all-state and partial-state (generalized cell-tocell) renormalizations. The first column $(f=1)$ corresponds to the conventional square-cell renormalization scheme.

\begin{tabular}{llllllll}
\hline & \multicolumn{7}{c}{$f$} \\
\cline { 2 - 7 }$b$ & & 1 & $\frac{1}{2}$ & $\frac{1}{3}$ & $\frac{1}{4}$ & $\frac{1}{5}$ & $\frac{1}{6}$ \\
\hline $6 / 1$ & $K^{*}$ & 0.4202 & & & & & \\
& $v$ & 0.7258 & & & & & \\
$5 / 1$ & $K^{*}$ & 0.4264 & 0.4198 & & & & \\
& $v$ & 0.7241 & 0.7300 & & & & \\
$4 / 1$ & $K^{*}$ & 0.4348 & 0.4286 & 0.4265 & & & \\
& $v$ & 0.7217 & 0.7271 & 0.7291 & & & \\
$3 / 1$ & $K^{*}$ & 0.4468 & 0.4422 & 0.4406 & 0.4398 & 0.4393 & 0.4390 \\
& $v$ & 0.7187 & 0.7225 & 0.7241 & 0.7249 & 0.7253 & 0.7257 \\
$3 / 2$ & $K^{*}$ & 0.4319 & 0.4235 & 0.4206 & 0.4191 & 0.4182 & 0.4176 \\
& $v$ & 0.7224 & 0.7322 & 0.7364 & 0.7386 & 0.7400 & 0.7408 \\
$4 / 3$ & $K^{*}$ & 0.4160 & 0.4072 & 0.4042 & & & \\
& $v$ & 0.7307 & 0.7420 & 0.7460 & & & \\
$5 / 4$ & $K^{*}$ & 0.4068 & 0.3989 & & & & \\
& $v$ & 0.7360 & 0.7466 & & & & \\
$6 / 5$ & $K^{*}$ & 0.4011 & & & & & \\
& $v$ & 0.7389 & & & & & \\
\hline
\end{tabular}

parametrized by the length factor $b$ and the area factor $f$. The critical point $K_{b f}^{*}$ and the critical exponent $\nu_{b f}$ are displayed for each point $(b, f)$. Note that the first column of table 7 , which corresponds to the special case $f=1$, lists the critical results obtained from the standard square-cell renormalization scheme [8]. The overall trend of the data in this table indicates that optimal results are obtained from renormalizations characterized by $(b, f) \rightarrow(1,0)$. The new and interesting finding is the effect of the rectangular area factor $f$ on the critical results. For fixed length factor $b$, both the critical point and the critical 
exponent improve as the area factor $f$ decreases. By fixed $b$ in table 7, we mean fixed horizontal size of the rectangular cells, i.e. fixed $m$ and $m^{\prime}$ for the mapping $n \times m \rightarrow n^{\prime} \times m^{\prime}$ where $b=m / m^{\prime}$ which is also equal to $n / n^{\prime}$. In rectangular renormalization, one has the freedom to choose not one, but two dimensions of the cell: the height and the width of the rectangle. The improvement of the critical results as $f \rightarrow 0$ corresponds to renormalizing taller rectangles of fixed width. In particular, since $n=m / f$ and $n^{\prime}=m^{\prime} / f$, the height of the rectangle scales as $1 / f$. Thus for the same $b$, the rectangle-to-rectangle renormalization $(f \neq 1)$ produces better results than the conventional square-to-square renormalization $(f=1)$. The faster convergence for rectangular lattices as $b \rightarrow 1$ or $b \rightarrow \infty$ is due to the fact that for a given lattice width, the rectangular area is larger than the square area by the factor $1 / f$. The other route to convergence for fixed $b$ is via $f \rightarrow 1$, which corresponds to using wider rectangles of fixed height. However, to produce a sufficient amount of data that follows this route, one needs to use lattices of much larger area than the relatively small lattice areas used in this study. Thus, from a practical point of view, the $f \rightarrow 0$ route is much easier to simulate than the $f \rightarrow 1$ route since there are exponentially fewer walks on tall rectangular lattices, such as $15 \times 3$, as compared with the number of walks on wide rectangular (limiting square) lattices, such as $15 \times 15$.

The difficult question of convergence for larger non-square lattices has been addressed in the phenomenological renormalization study of self-avoiding walks on infinite strips [13]. For the special case of infinitely tall rectangles and a scale factor of $b=m /(m-1)$, this study finds an overall rapid convergence to the exact values as $b \rightarrow 1$, with monotonic convergence for strips with periodic boundary conditions. Thus it appears that the finitesize rectangles used in rectangular renormalization produce results that extrapolate to the exact values for a sequence of larger and larger rectangles. In contrast to this infinite-lattice study, we systematically investigate the effect of moving away from the standard square-cell renormalization to demonstrate the existence of simple and accurate finite-lattice schemes.

We are not aware of other small-cell renormalization calculations that have consistently achieved the level of accuracy of rectangular renormalization. For each renormalization, characterized by a different area factor $f$, we were able to obtain a value for the critical exponent in the range $0.74<v<0.75$ from relatively simple calculations. Of particular note are the renormalizations corresponding to $(b, f)=\left(\frac{4}{3}, \frac{1}{3}\right)$ and $\left(\frac{5}{4}, \frac{1}{2}\right)$ which yield $K^{*}=0.4042, v=0.7460$ and $K^{*}=0.3989, v=0.7466$, respectively.

To illustrate this simplicity and accuracy of rectangular renormalization, we compare our results with the best results obtained from other finite-lattice renormalization studies. The cumulant-like, majority-rule, multiple-cell renormalization of [10] finds a result of $K^{*}=0.309, \nu=0.712$ from a second-order map consisting of five coupled equations containing five coupling constants corresponding to different step weights with memory. The site-decimation, cluster renormalization of [14] finds a result of $K^{*}=0.4469, v=$ 0.7386 using a two-step approximation involving eight coupling weights, and a result of $K^{*}=0.3780, v=0.7538$ using a three-step approximation with twelve coupling weights. For the single-cell renormalization results, corresponding to the special case $f=1$, see [8]. Some of the best results obtained from this square-cell renormalization study are $K^{*}=0.4088, v=0.7449$ from a $6 \times 6 \rightarrow 1 \times 1$ transfer matrix analysis; $K^{*}=0.3759, v=0.7443$ using $5 \times 5 \rightarrow 4 \times 4$ toroidal cells; $K^{*}=0.3807, v=0.7270$ from a Monte Carlo simulation on $150 \times 150 \rightarrow 1 \times 1$ square cells. Note that the complexity of all the above renormalization schemes is significantly greater than that of rectangular renormalization. For a similar level of complexity, one should note the largest, pure and simple cell-to-cell renormalization that has been done, $6 \times 6 \rightarrow 5 \times 5$, gives a result of $K^{*}=0.4011, \nu=0.7389[8]$. We have extended this square cell $(f=1)$ result by 
performing a $7 \times 7 \rightarrow 6 \times 6$ renormalization and find $K^{*}=0.3971, v=0.7402$. This should be compared with our $10 \times 5 \rightarrow 8 \times 4$ result of $K^{*}=0.3989, v=0.7466$, since both of these calculations involve similar computation time. It is interesting to note that the $7 \times 7 \rightarrow 6 \times 6$ square cell computation is slightly more complex ( $2 \%$ more computation time) than the $10 \times 5 \rightarrow 8 \times 4$ rectangular cell computation, in spite of the fact that the $10 \times 5=50$ total site area is slightly larger than the $7 \times 7=49$ total site area. This reflects the asymmetry in the entropy of the spanning walks as it depends on the horizontal and vertical dimensions of the finite lattice.. Thus, for a similar level of computational complexity (similar cell area), the pure rectangle-to-rectangle renormalization gives a significantly better critical exponent than the pure square-to-square renormalization.

\section{Discussion}

Rectangular renormalization is a generalized renormalization. It is characterized by two scale factors: the length scaling factor $b \equiv \ell^{\prime} / \ell$, and the area fraction factor $f \equiv \omega / \Omega$. Parametrized by $b$ and $f$, there exists a family of renormalizations, $1<b<\infty$ and $0<f \leqslant 1$, that include some of the standard schemes as special cases. Geometrically, rectangular renormalization represents a mapping between spanning states on rectangular sublattices. Algebraically, it represents a partial partition function invariance.

The practical significance of rectangular renormalization lies in its simplicity and accuracy. It seems to be a high-efficiency renormalization scheme in that it produces the most accurate results for a given amount of calculation. For the self-avoiding random walk problem, the results are comparable to, and in most cases better than results obtained from previous renormalization studies based on additional rules, higher-order approximations and/or more complex computations. As with square renormalization, there is no a priori justification for the use of the rectangular cell geometry of rectangular renormalization. However, given the rectangular results, it appears that large square lattices and intricate mapping rules are not necessary to achieve reliable results.

A new discovery is the effect of the rectangular area fraction on the critical descriptors. Unlike square renormalization (parametrized by $b$ ), rectangular renormalization (parametrized by $b$ and $f$ ) allows one independent control over both the vertical and the horizontal dimensions of the rectangular lattice. For fixed $b$, there exist two distinct optimal paths in $(b, f)$ parameter space for which monotonic improvement occurs: the $f \rightarrow 0$ path. corresponding to increasing the height of rectangles of fixed width, i.e. $(m / f) \times m \rightarrow$ $\left(m^{\prime} / f\right) \times m^{\prime}$, and the $f \rightarrow 1$ path, corresponding to increasing the width of rectangles of fixed height, i.e. $n \times f n \rightarrow n^{\prime} \times f n^{\prime}$. For each of these paths, the improvement is due to increasing one of the dimensions of the rectangle. The limiting critical values $K_{b 0}^{*}, v_{b 0}$ for $f=0$, and $K_{b 1}^{*}, v_{b 1}$ for $f=1$, represent optimal values for a given $b$ that become more exact as $b$ approaches 1 or $\infty$. For fixed $f$, we find that optimal renormalization occurs in the limit $b \rightarrow 1$ or $b \rightarrow \infty$, which is a generalization of the well known square-lattice $(f=1)$ renormalization behaviour. This behaviour in the $b-f$ plane provides insight into the basic anatomy of renormalization and practical criteria for designing a reliable approximation.

The accurate results that emerge from rather modest calculations are due to the effective large-lattice nature of rectangular renormalization. The renormalization of the whole $\Omega$ lattice is accomplished by focusing on the smaller $\omega$-lattice. More specifically, although the actual calculation occurs on relatively small rectangular sub-cells: $n \times f n \rightarrow n^{\prime} \times f n^{\prime}$, i.e. $12 \times 4 \rightarrow 9 \times 3$, the corresponding renormalization map refers to the original large square cells: $n \times n \rightarrow n^{\prime} \times n^{\prime}$, i.e. $12 \times 12 \rightarrow 9 \times 9$, from which the rectangles are formed. The subset of walks that span a rectangular sub-lattice provide a realistic representation 
of a class of waiks embedded on the larger square lattice that project correctly under the renormalization. In other words, walks which span a rectangle seem to have the correct macroscopic connectivity and relevant topological information to produce a renormalization map that is a good approximation to the exact (infinite square lattice) renormalization map. This indicates that the partial partition function defined on the rectangular cell contains the crucial coarse-grained, renormalization information. The whole partition function defined on the square cell is not necessary. This is perfectly consistent with the fundamental principles of the renormalization-group theory. According to these exact principles, although a renormalization map must leave the whole partition function invariant on all of state space (see equation (2.3)), the map itself is rigorously defined only between subsets of state space (see equation (2.1)). Walks confined to a rectangular sub-cell seem to provide a physically correct subset.

In a certain sense, our generalized renormalization scheme can be viewed as a hybrid scheme composed of the successful elements that characterize other real-space renormalization approximations. We have already discussed how rectangular renormalization, being based on a partial partition function, incorporates features from both the single-state and the all-state renormalization schemes. Other hybrid features are localization and spanning. In rectangular renormalization, the renormalization takes place between localized walks confined to a rectangular region within a single cell. The extent of the localization, as measured by the size of the rectangle, is determined by the area fraction $f$. This localization is characteristic of the cluster renormalization schemes that include the site decimation $[9,14]$ and the cumulant-like, multiple-cell [10] schemes. In rectangular renormalization, the cluster is simply a rectangle. The other feature of rectangular renormalization, that of spanning across a single cell, is characteristic of the well known cellular renormalization schemes [8].

There are several natural extensions of rectangular renormalization as presented here. These include application to other critical phenomena, non-rectangular sub-cell geometries, three-dimensional 'cylindrical renormalization', perturbation into the forbidden zone, and analytic scaling in $f$ and $b$. Preliminary studies of incommensurate rectangles $\left(f \neq f^{\prime}\right)$ indicate the expected result that the rectangular area should be preserved under the renormalization. It is hoped that rectangular renormalization will provide further insight into the nature of real-space renormalization and critical phenomena.

\section{References}

[1] Burkhardt T W and van Leeuwen J M J 1982 Real-Space Renormalization ed T W Burkhardt and J M J van Leeuwen (Berlin: Springer)

[2] Cirillo E N M and Gonnella G 1995 J. Phys. A: Math. Gen. 28867

[3] Vespignani A, Zapperi S and Pietronero L 1995 Phys. Rev. E 511711

[4] Stanley H E, Reynolds P J, Redner S and Family F 1982 Real-Space Renormalization ed T W Burkhardt and $\mathrm{J} M \mathrm{~J}$ van Leeuwen (Berlin: Springer)

[5] Martinelli F and Olivieri E 1995 J. Stat. Phys. 7925

[6] Ziff R M 1992 Phys. Rev. Lett. 692670

[7] Aharony A and Hovi J-P 1994 Phys. Rev. Lett. 721941

[8] Redner S and Reynolds P J 1981 J. Phys. A: Math. Gen. 142679

[9] Shapiro B 1978 J. Phys. C: Solid State Phys. 112829

[10] Napiorkowski M, Hauge E H and Hemmer P C 1979 Phys. Lett. 72A 193

[II] Conway A R, Enting I G and Guttmann A J 1993 J. Phys. A: Math. Gen. 261519

[12] Nienhuis B 1987 Phase Transitions and Critical Phenomena vol 11 ed C Domb and I L Lebowitz (New York: Academic)

[13] Derrida B 1981 J. Phys. A: Math. Gen. 14 L5

[14] Malakis A 1980 Physica 104A 427 